\title{
A Comparative Study of Routing Protocols in e-Health Applications for Heterogeneous Interfaced Mobile Ad-Hoc Grid
}

\author{
S. Jan, I.A. Shah and I. Khan \\ University of Engineering and Technology, Mardan Campus \\ Mardan, Khyber Pakhtunkhwa, Pakistan.
}

\begin{abstract}
Mobile computing has become an indispensable way of life in recent years. Recent advancement in Mobile phones and other handheld devices have enabled them of using various types of networks, which were meant for computers only. The integration of such devices into heterogeneous grids has emerged new research challenges. Different types of devices with heterogeneous interfaces and computational powers require versatile mechanisms to cope with various types of applications and situations. This paper presents the performance analysis of reactive and proactive routing protocols used for mobile ad-hoc grids in e-health applications. The performance of four protocols is analyzed in terms of routing load and response time. Further, the feasibility of heterogeneous interfaces in mobile ad-hoc grid is analyzed in terms of energy consumption.
\end{abstract}

\section{Keywords}

Mobile Grid, Routing Protocols, E-Health, Ad-hoc Grid, Mobility, Heterogeneous Grid

\section{INTRODUCTION}

In Grid computing environments, the resources of all connected computing devices can be utilized in pervasive manner. These devices can be personal computers, laptops, mobile phones, PDAs or other hand-held computing devices. However, more extensive research is required to fully utilize the advantages of grid computing. For this purpose, the traditional network and application technologies should be well-suited for all types of computing devices. In mobile adhoc girds, all the unused resources of devices can be shared to process a specific task assigned in such environments. These resource limited devices may have different limitations to process some tasks required by computationally expensive applications. Scientists and researchers have suggested different mechanisms to overcome these challenges like [1] has investigated and proposed a mobile agent paradigm to develop a middleware. Their system deals with underlying existing details and allows mobile users to access the grid resources transparently.

Mobile Ad-hoc Networks (MANETs) [2] consists of mobile wireless nodes, where each node plays the role of source and destination as well relay data for others in a multi-hop fashion. Since the deployment of MANETs needs no existing infrastructure, therefore it has got its applications in scenarios having emergency situations such as natural disasters and military operations. Despite their easy deployment, MANETs have some associated inherent issues. First, the power consumption as nodes run on batteries and the design of any protocol should address this important issue primarily. Second, the capacity of network is constrained by the number of nodes due to the inherent nature of wireless medium and the multi-hop relying. These issues need to be addressed effeminately while developing MANETs protocols for an optimized real world system.

MANETs nodes usually experience high mobility and the topological changes are very likely to take place frequently. The rapid topological changes recurrently results in a wireless domain where the dynamics of the network should be accurately captured to know the location of each node. This challenging task demands an efficient routing protocol to capture these time varying changes, accurately. While in pure MANETs, each node can be a source or destination for data as well as a forwarding router for others. The integration of grid system, however, changes the scenario to impound the source destination nodes to the limitation of a few nodes. An architecture proposed by [3] identified many problems which arise from deploying grid computing over ad-hoc architecture. In their proposed architecture, service discovery and job scheduling components work together with ad-hoc routing protocols and some of the grid functionalities are designed in distributed fashion through ad-hoc networking protocol stack.

Due to the progression in technology, recent mobile devices are equipped with multiple heterogeneous interfaces. This capability provides many choices to the device for accessing the network. In MANETs, for example, each node can have multiple interfaces where two communicating nodes can select the appropriate one for data transfer in a specific communication session. This added advantage not only provides flexibility in terms of connectivity but ease to optimize the system with the objectivity of power consumption and achievable data rates. While routing plays a vital role in the performance of MANETs due to the high mobile nature of nodes and the dynamics of the topological changes, power consumption can be reduced through many other methods, including the use of appropriate interfaces which need low power for transmission and reception, while satisfying the required data rate by the specific communication session. For wireless networks like MANETs, where nodes are highly mobile, runs on non permanent power supplies and having bandwidth limitations, routing becomes much more imperative to be considered as a key factor. This paper is the extension of one of our previous work [4], where we addressed the issue of selecting an appropriate routing protocol in the e-health grid over an Ad-hoc Wireless Network.

The rest of this paper is organized as follow: In Section 2, a brief discussion is given about relevant work. Section 3 describes our application scenario and routing protocols for MANETs. In Section 4 we present extensive simulation study and propose the best routing protocol for the e-health grid application under consideration. In Section 5, we discuss the proposed model for a heterogeneous interfaced Ad-hoc grid and the selection criteria for selecting appropriate interface, given the power/data rate thresholds. In this section we also 
analyze the advantages of the proposed system in terms of energy consumption. Finally, Section 6 concludes our work and gives direction for future study.

\section{RELATED WORK}

A thorough analysis of the performance of several routing protocols has been addressed in many research studies with the consideration of different applications and scenarios. A scenario based performance analysis for MANETs is presented in [5]. In their study, mobility metric is taken as a parameter for judging the protocols performance with different scenarios setup. Their analysis has been presented in the form of mobility metric quantification of nodes' movement relevant to MANETs. Reactive behavior of routing protocols to network dynamics, topology changes and successful data delivery ratio has been extensively studied in [6]. While they have analyzed the protocols' performance under different conditions, their work lacks the consideration of data communication in real world scenarios. Authors in [7] have carried out the performance evaluation study for a set of on-demand routing protocols, where packet delivery and routing overheads are taken for comparison using CBR traffic sources. Comparison of various routing metrics with ETX for DSR protocols has been carried out in [8], where ETX is proposed as the best metric to capture the quality of links. In [9], authors have studied the performance of routing protocols based on the power consumption.

Adding multiple interfaces to mobile nodes has been studied in different contexts. Several studies $[10,11,12,13,14]$ focuse on the channel assignment problem to multiple radios/interfaces of nodes, where the interfaces belong to the same technological standard e-g IEEE 802.11. Some research work has further extended to include the heterogeneity across multiple radios of the nodes. For example in [15], authors have addressed the co-existence of heterogeneous interfaces where scheduling, routing and channel assignment is solved as an optimization problem. They have addressed the heterogeneity of interfaces belonging to same technology i.e. coexistence of IEEE 802.11a, 802.11b and 802.11g on the same mobile node. Integration of interfaces belonging to totally different set of technologies is studied in [16], where architecture is proposed and the benefits of heterogeneous network interfaces have been highlighted by formulating it as an optimization problem.

In one of the previous study [17], we have proposed an integrated architecture based on radios belonging to three different technologies. The analytical results proved that the throughput enhancement is possible and an interface switch mechanism has been proposed based on a pre-defined threshold. We have compared reactive and proactive routing protocols in mobile Ad-hoc grid environment on the basis of response time and routing overhead in this article. Furthermore, the performance of overall system is compared in terms of power saved when heterogeneous interfaces are used. Compared to other related research, our study is different as we have integrated a mobile grid paradigm in heterogeneous interfaced MANETs.

\section{MANETS ROUTING PROTOCOLS}

Routing protocols for MANETs can be broadly categorized into two classes, proactive or table driven and reactive or on demand. The main difference between these two classes of protocols is how the routing information is maintained by the individual nodes. Routing determines the end to end path from a source node to a destination node. In the case of Proactive routing protocols, each node maintains routing tables and are periodically updates it. These routing tables on each node contain fresh routes to all other nodes in the network. Thus, each node knows the path to all other nodes in the network. The advantage is fast response time at the cost of higher routing overhead. OLSR [18] and DSDV [19] belong to this class.

In the case of Reactive routing protocols, each node does not need to maintain the routing information. These protocols are called on demand as the routing path is determined from source to destination prior to data session in the following manner. For a data session between source and destination nodes $(\mathrm{A}, \mathrm{Z})$, the route request is initiated by the source $\mathrm{A}$ with a route discovery packet broadcast. Each neighbor of A receives this packet and rebroadcasts it to their neighbors until it reaches to the destination $\mathrm{Z}$. The source $\mathrm{A}$ is notified of this route in reverse. Since routing tables are not maintained at the nodes, reactive routing protocols have lower processing overhead, less memory requirements, less power consumption and saves bandwidth. The main disadvantage of reactive routing protocols is the initial latency involved during route discovery phase. AODV [20], DSR [21] and TORA [22] are protocols which fall in this category. Following, we have summarized all those reactive and proactive routing protocols which are used in our simulations.

A candidate proactive routing protocol is Optimized Link State Routing (OLSR) which is an optimized version of link state routing protocol. OLSR differs from the common link state algorithm by minimizing the flood effect of control messages for links dissemination in the network. Only special nodes, called Multiple Relays (MPR) are responsible to diffuse control traffic in the network. OLSR protocol works in four steps. First, detection of neighbor nodes and two hop neighbors with "HELLO" messages. In the second step, MPR is selected by each node which is a subset of those one hop neighboring nodes having bi-directional links to the two hop neighbors of this node. In step three. each node broadcasts Topology Control (TC) messages, which contains information of the multipoint relays selectors of these sending nodes. Information from TC messages, about the multipoint relays selectors of all other nodes in the network, is fed by each node in the topology table. In the final step, routing tables are computed from topology table which was calculated in the previous step.

Geographical Routing Protocol (GRP) [23] is one of the implementation of geographical assisted routing protocols for example, GeoCast [24] and Distance Routing Effect Algorithm for Mobility (DREAM) [25]. Instead of logical addresses, geographical locations in terms of $\mathrm{x}, \mathrm{y}$ coordinates from Geographical Position System (GPS) are used for updates in the routing tables. The Ad-hoc On-Demand Distance Vector (AODV) is another important reactive routing protocol which consumes less memory, processing power, bandwidth and energy [20]. If a source node wants to send some data to a destination node which is previously unknown, a route discovery is initiated by the source node with a broadcast Route REQuest (RREQ) message to its immediate neighbors. The same request message is rebroadcasted by the source node's neighbors to their neighbors. This process continues till the request reaches the destination. The important field in the RREQ packet is the Destination Sequence Number, which avoids loops in the routing paths.

Upon reaching the destination, a unicast Route REPly (RREP) packet is sent back to the source node by the destination through its immediate neighbor from which it 
received the first RREQ. Dynamic Source Routing (DSR) uses the source routing algorithm in which a query packet is initiated by the source and the address of each intermediate nodes it transverses are recorded inside the query packet's header. Upon reaching the destination, the list of address learnt through query packet is reversed to enable the packet to reach the source while providing it the path at the same time. Each data packet carries the complete route to the destination in its header. DSR nodes keep the learned routes in its cache for efficient route discovery. Source routing enables DSR nodes to keep multiple routes to a destination. In case of link failure, alternate path is searched from the cache. If no such alternate route exists in cache, a new search is initialized. The path information contained in the packet header makes the detection of loops efficient [2].

\subsection{Application Scenario}

To demonstrate the selection of best routing protocol in situation of emergency, a scenario is presented in Figure-1, considering wireless Ad-hoc network as a communication base. The Ad-hoc grid has been further extended by enabling multiple heterogeneous interfaces on each node. The performance is investigated in terms of energy consumption taking homogenous Ad-hoc grid as a benchmark. As presented in Figure 1, on frontline positions, a soldier is in need of medical treatment immediately. A complete database is available at the base camp about the medical history of every soldier. There is a medical-software application simulator which takes the current status of the patient as input and requires his medical history from data base to simulate the best treatment needed in emergency situations. At the time of emergency, some of the soldiers have access to mobile phones or laptops running this application. As the nearby soldier with a mobile phone realizes the situation, he immediately communicates the situation to his neighboring node requesting to simulate and send back the results because his device is not capable of executing the complete application. When the soldier with a laptop, receives the request, it takes the current status and history and the scheduler on his laptop will distribute the simulation tasks to neighboring devices as his laptop will take too long to complete the execution. Finally, after getting back the results from the executing nodes, he sends back the result/response to requesting node on best available path for further processing of the requested information.

\section{SIMULATION SET AND ANALYSIS}

We analyzed the performance of proactive and reactive protocols using two different scenarios which include small network with low mobility and large network with high mobility, each scenario having different number of execution hosts. The network simulator, OPNET modeler version 14.5 was used for simulation of all our scenarios. The results were analyzed in terms of routing overhead and response time of the grid application. Routing load refers to total number of packets generated by all the nodes in the network for establishing end-to-end routes between submitting hosts (sources) and execution hosts (destinations). The metric response time refers to the time taken between sending a request by a submitting host to an execution host and receiving back the response/result. Response time can be expressed mathematically as:

$$
R_{t}=E_{t}+N_{t}
$$

Where $R_{t}$ is the response time, $E_{t}$ is the execution time of a query which depends upon the number of execution hosts, their processing power, memory and other resources and $N t$ is total network delay came across by all data packets exchanged between execution host, submitting hosts and resource manager (database server) during a request and response session. While keeping $E_{t}$ of all the execution hosts same, $R_{t}$ totally depends upon $N_{t}$ which varies with topological changes due to mobility of normal ad-hoc nodes, execution hosts as well as submitting hosts. We have analyzed the behavior of routing protocols in terms of response-time $R_{t}$ by varying number of execution hosts, and hence $E_{t}$.

\subsection{Scenario 1:}

In scenario-1, a network of 50 ad-hoc nodes was deployed in which 5 were submission hosts and 4 were execution nodes. The network was configured with low mobility trajectories with a pause time of 30 seconds after each segment of length $50 \mathrm{~m}$ for each node. A group mobility mode as in [26] was applied to the ad-hoc nodes. Group mobility best suits to the application scenario as outlined in section 3.1. The speed of nodes was kept $10 \mathrm{~km} /$ hour in specific direction across $500 \mathrm{mx} 500 \mathrm{~m}$ area. The same ad-hoc mobile grid scenario was used for the analysis of OLSR, GRP, AODV and DSR. As depicted in Figure 2, GRP generated least routing traffic averaging 500 packets per second as compared to OLSR which generated 1300 packets per second on average, hence a performance difference of $37.92 \%$. A high routing overhead by GRP at the beginning of simulation is due to the initial flooding technique used by this protocol. The protocol comes to a stable state after 180 seconds of simulation time once the flooding is completed. The mobility of nodes had very minor effect on GRP's performance because the expiry time was set to 10 seconds. The only routing overhead produced by GRP is the normal HELLO messages. Overhead caused by flooding updates is reduced by a technique called fuzzy routing in which the nodes which need to know about the position updates receive the flood.

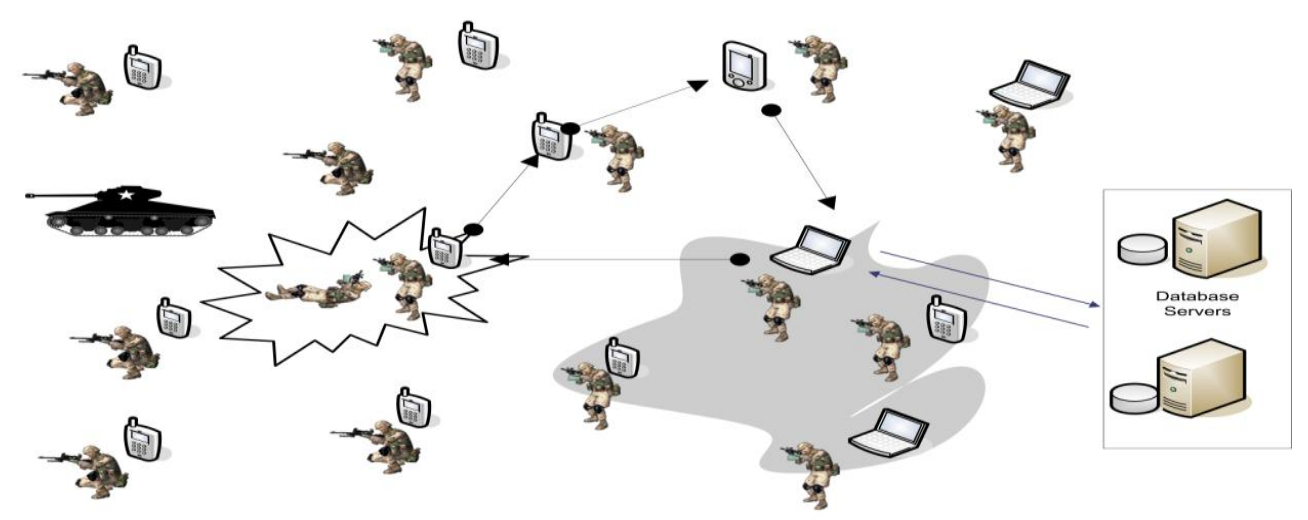

Fig 1: Application scenario 
Among the reactive routing protocols, DSR outperformed AODV by producing $30.43 \%$ less routing traffic. This is because less route request and route reply messages are produced by DSR as compared to AODV during the route construction phase. A very minor increase in routing overhead was observed for all the routing protocols when the number of execution hosts was increased to 6 . This is because the execution hosts has low mobility in ad-hoc grid. DSR proved to be more efficient by producing low routing overhead overall followed by GRP while OLSR produced high overhead routing packets in Scenario 1. When the simulation results were analyzed for the response time metric, both proactive routing protocols performed almost the same with very little difference of $9.4 \%$ by which OLSR outperformed GRP. This is because OLSR takes a very prompt action to the link failures and updates the routing tables very frequently as compared to GRP.

Among the reactive protocols, DSR outperformed AODV with a $12.38 \%$ quick response time as shown in figure 3 . The reason is DSR's capability to store previous discovered routes in its caches which enables DSR discovery function less time consuming. When the same configuration was tested for response time by increasing the number of execution hosts to 6 , a very small improvement was observed for all the protocols under test. The reason for so small improvement in the response time is due to the fact that when an increase in the number of executing hosts decreases Et which further decreases Rt. In a conclusion, for a network having low mobility, small size and low load, OLSR proved to be the best routing protocol for grid application's response time followed by GRP while AODV proved to be the worst.

\subsection{Scenario 2:}

In scenario 2, a network of 104 mobile ad-hoc nodes was deployed over a square area of $1000 \mathrm{~m} \times 1000 \mathrm{~m}$ as a mobile adhoc grid. The number of grid submitting hosts was set to 10 and an evaluation was carried out for 5 and 12 execution hosts in the same scenario separately. The network was configured with high mobility trajectories with a pause time of 5 seconds after each segment of length $70 \mathrm{~m}$ for each node. A group mobility mode as in [26] was applied to the ad-hoc nodes by dividing the whole network into 6 groups. The speed of nodes was kept $20 \mathrm{~km} /$ hour in specific direction across $1000 \mathrm{mx} 1000 \mathrm{~m}$ area. All the four routing protocols under consideration were analyzed for routing load produced by each and the response time to the grid application queries. Figure 4 shows that among the proactive protocols, GRP performed better than OLSR comparatively by producing less routing overhead.

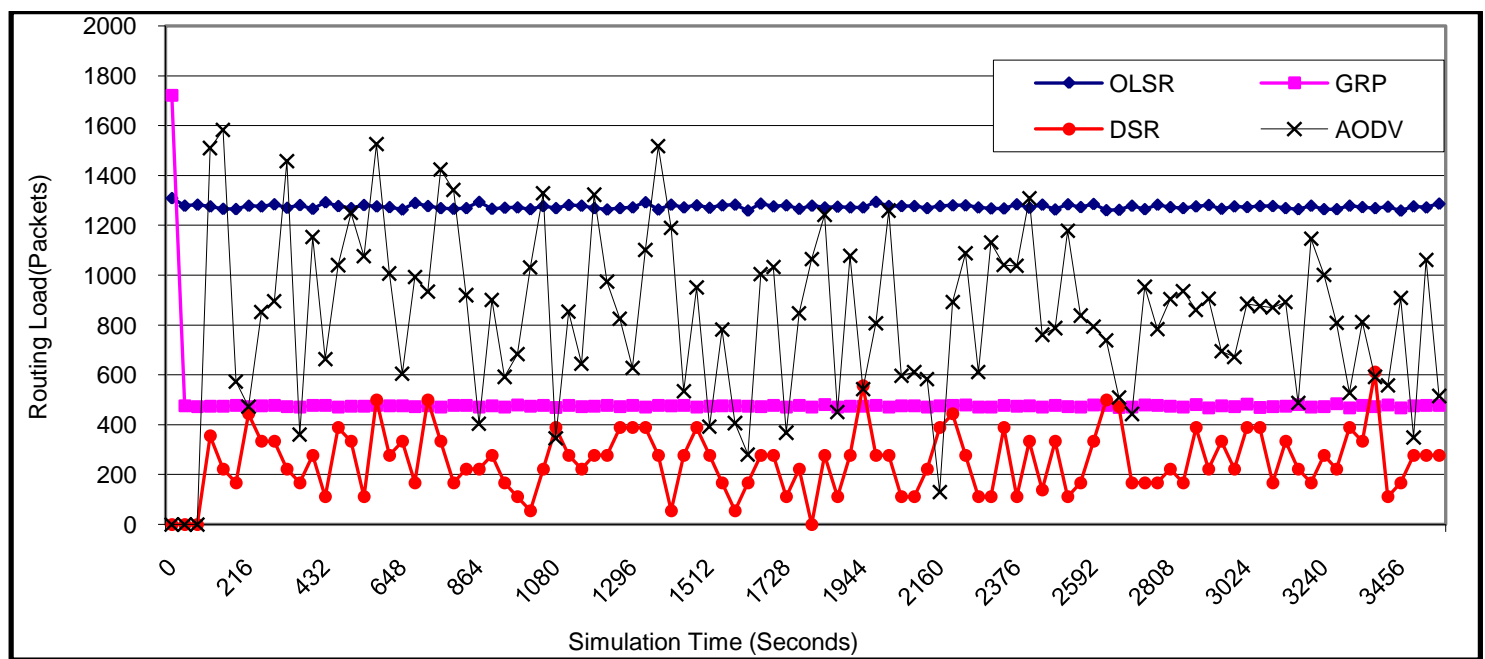

Fig 2: Routing load of small network with low mobility and 4 execution host

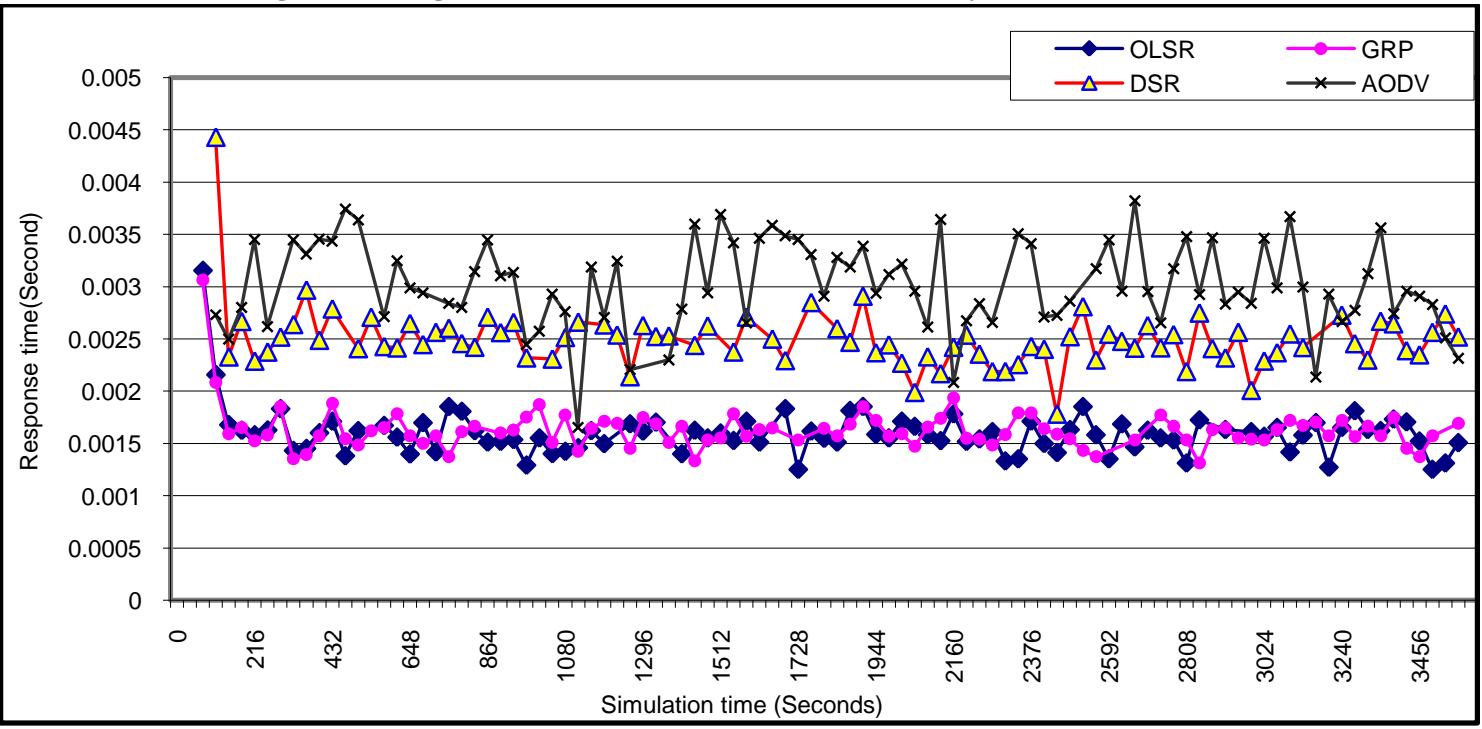

Fig 3: Response time of small network with low mobility 
An abrupt spike at the beginning of simulation by GRP routing overhead can be seen in figure 4 . This high overhead for a short time is due to the high flooding mechanism used in GRP during route the route initialization phase. Among the reactive protocols, DSR produced less routing overhead packets (4678) as compared to AODV which produced 5935 packets on average. The basic reason is that the high mobility of nodes results in more frequent link failures. Since AODV produces more RREQ, RREP packets as compared to DSR and hence more routing overhead. Overall, when the network is large and nodes are highly mobile GRP performs better among all protocols in terms of routing overhead. This is followed by DSR and OLSR, respectively. For a high mobility profile and large network, OLSR response time is better among the reactive routing protocols due to the same quick response time to link failure. DSR again outperformed AODV with a huge gap because of its capability of storing routes in its caches.

The increase of execution hosts has an impact on the response time for all protocols, as can be seen in figure 6 . When the number of execution hosts is increased from 5 to 12 , there is an improvement in response time for all protocols which can be seen in Figure 6. OLSR response time is increased by $10.3 \%$ on average while GRP shows an increase of $9.2 \%$. Reactive protocols show very little improvement in response time ranging from $8.34 \%$ for DSR to $7.3 \%$ for AODV. In conclusion, for a profile with high mobility, high load and large size, OLSR performed better in terms of response time among all the proactive and reactive routing protocols. This was followed by DSR, while GRP being the worst.

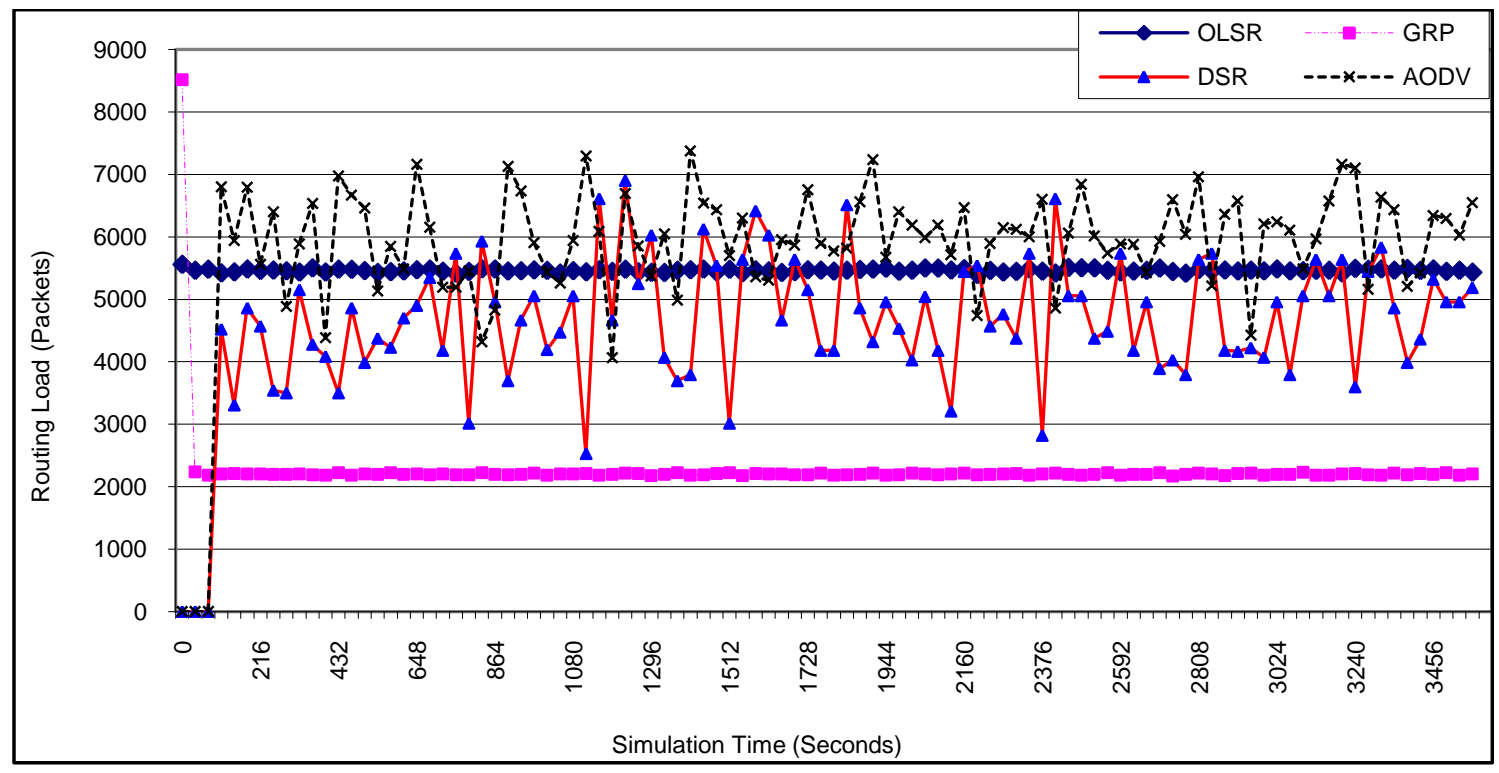

Fig 4: Routing load (large network, high mobility) with 5 execution hosts

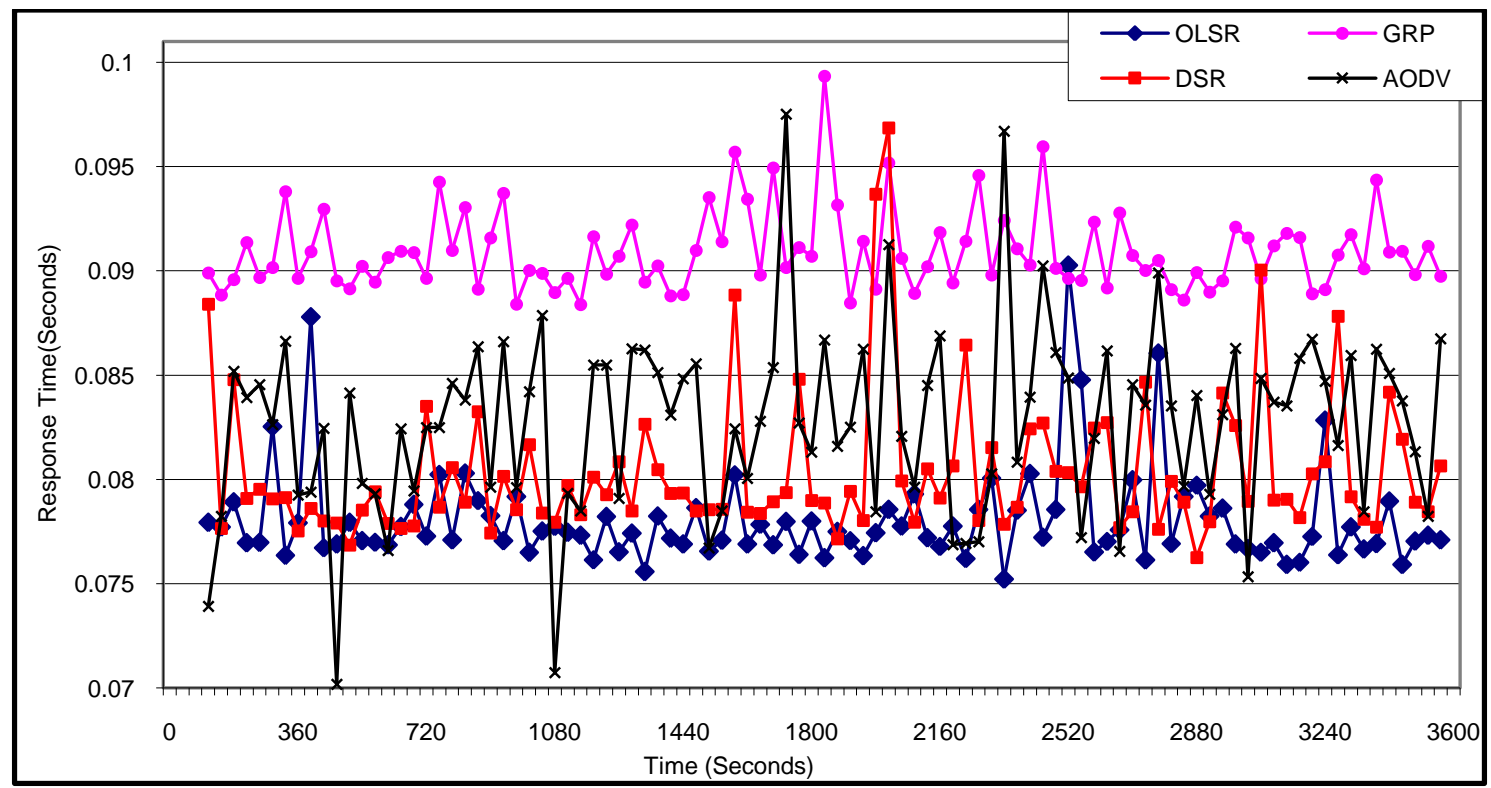

Fig 5: Response time (large network, high mobility) and 5 execution hosts 


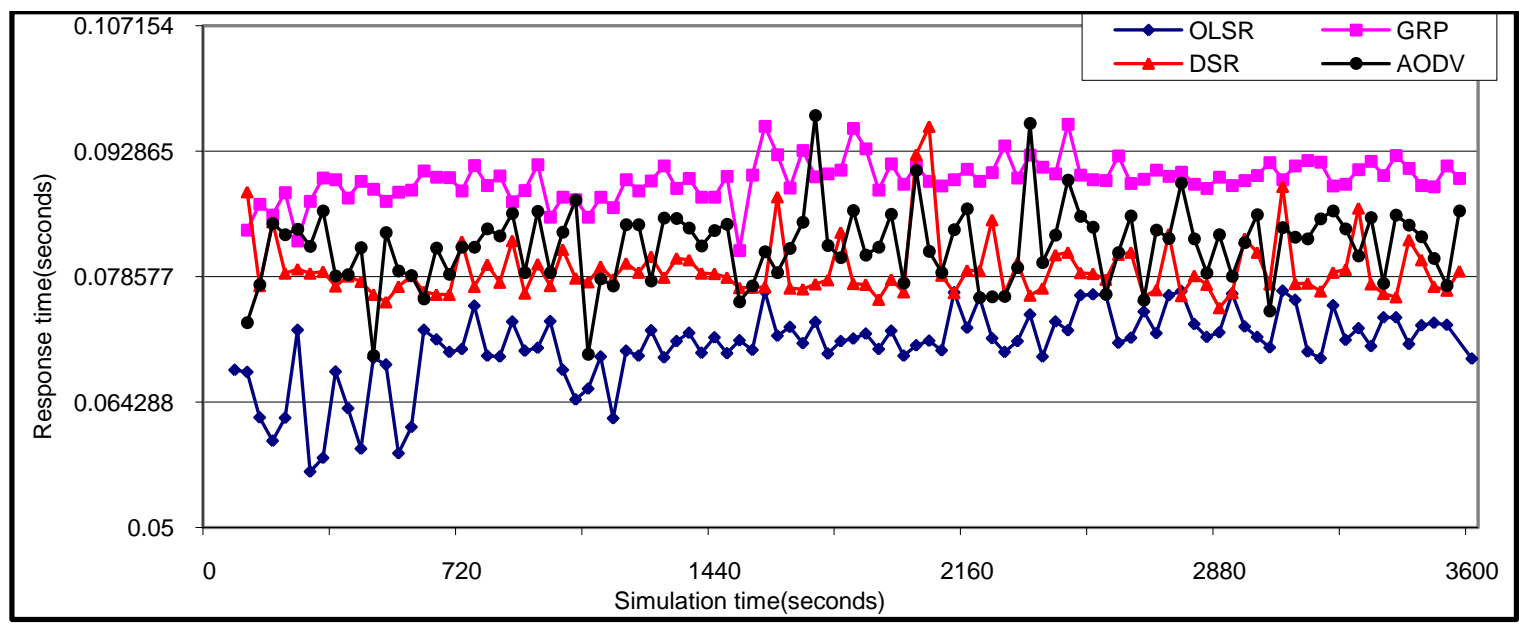

Fig 6: Response time (large network. high mobilitv) and 15 execution hosts

\section{HETEROGENOUS INTERFACES CO- EXISTENCE IN AD-HOC MOBILE GRID}

\subsection{System Model}

In standard MANETs, each node has one interface/radio per node and it is necessary that all nodes' interfaces follow the same standard at Medium Access Control (MAC) layer in order to communicate with each others, e-g all nodes have IEEE $802.11 \mathrm{a} / \mathrm{b} / \mathrm{g}$ interfaces. Adding multiple interfaces can increase the network throughput and connectivity as multiple parallel communication sessions can be achieved by tuning set of communicating radios to orthogonal channels. Equipping node with multiple radios, however, has some disadvantages as well. For example, MANETs are power constrained networks and adding extra interfaces to the nodes means consumption of more energy which scales up with the number of radios. In this paper, we integrate the heterogeneous radios on the MANETs nodes as proposed in one of our previous work [17]. We, however, keep our implementation limited to the case of IEEE 802.11 [27] Wi-Fi and IEEE 80.15 [28] Bluetooth interfaces only. As shown in Figure 7, a wireless Ad-Hoc network is shown with each node having two radios. Submission and execution nodes of the grid are part of the Ad-hoc network. Upon the submission of query, the data is passed on to the execution nodes in multi-hop fashion exploiting the rely nature of MANETs. The decision of interface selection between two adjacent nodes is based on two parameters. First each node evaluates the bandwidth requirements of the requested query/data and the remaining

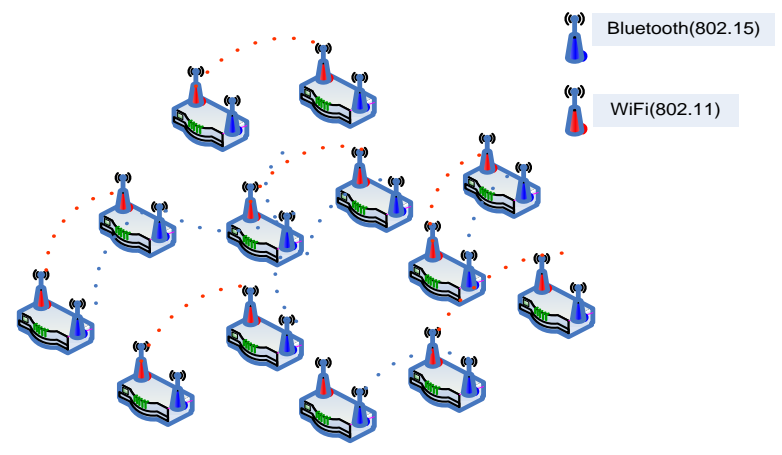

Fig 7: Mobile Ad-hoc Grid with heterogeneous interfaces battery life of the devices. If the data rate requirements are met by both the underlying technologies of specific required for the data session. The simple algorithm is presented in Figure 8 to explain the interface selection of a communicating nodes pair(n1, n2) for a specific communication session.

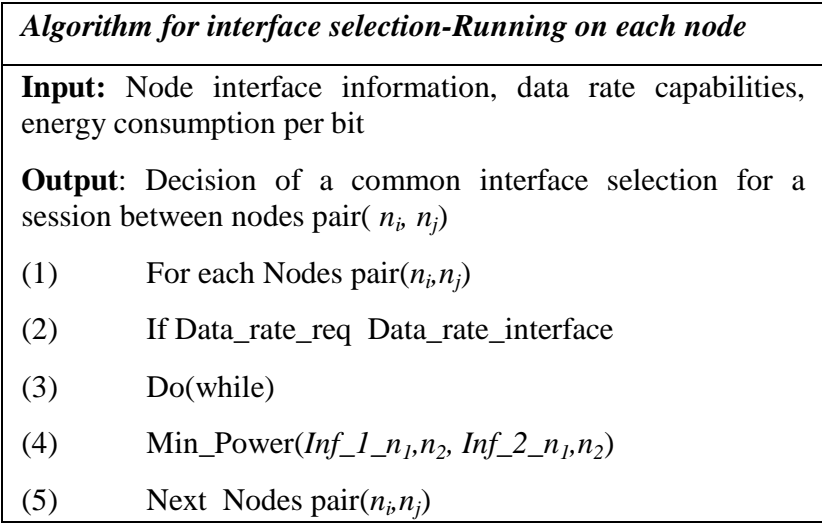

Fig 8: Algorithm for interface selection

Where Inf $1{ }_{-} n_{1}, n_{2}$ is the set of interfaces of the nodes pair $\left(n_{l}\right.$, $n_{2}$ ) belonging to technology 1(e.g IEEE 802.11) and Inf_2_ $n_{1}, n_{2}$ is the set of interfaces between the nodes pair belonging to technology 2(e.g IEEE 802.15).

\subsection{Simulation Setup and Results}

The proposed algorithm was implemented in OPNET version 14.5. A generic layer was defined between MAC and Network (Routing) layers for making the decision for the interface selection, as shown in Figure 9. The core algorithm was implemented inside the generic interface selection layer.

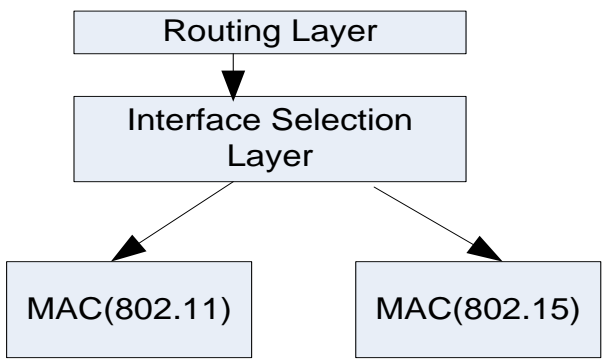

Fig 9: Generic Interface Selection Layer 


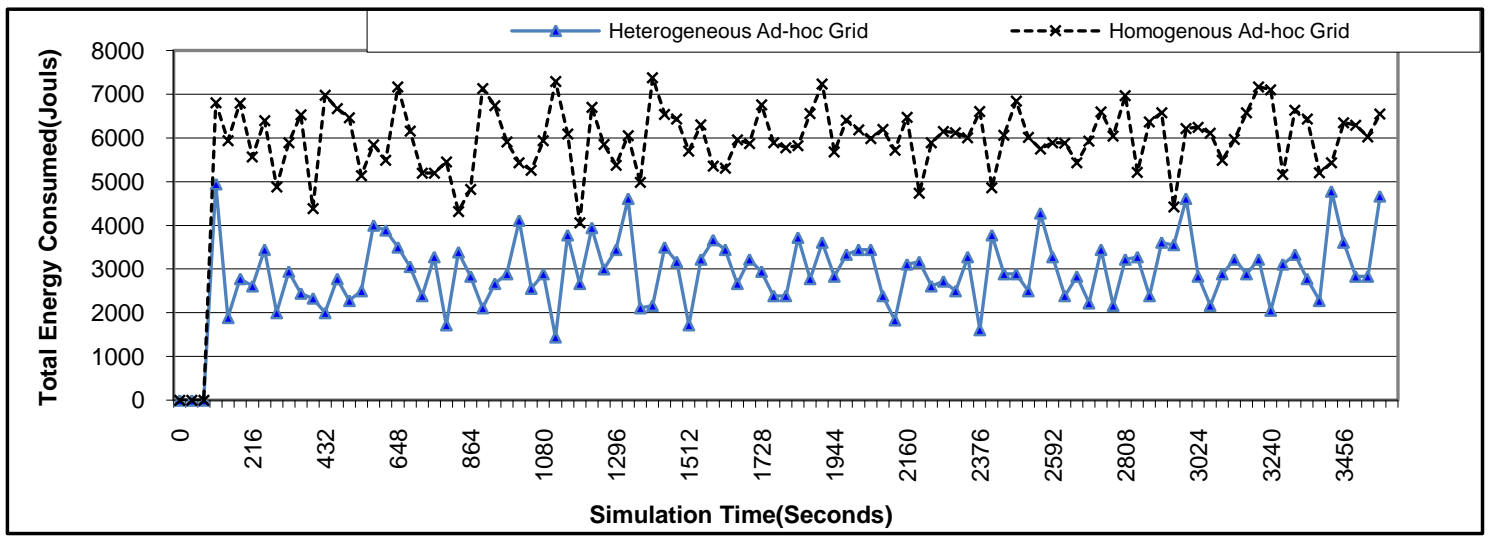

Fig 10: Total System Energy consumption (medium network, moderate group mobility) with 5 submissions and 4 execution hosts

\subsubsection{Scenario 3:}

The performance evaluation was carried out for a medium network consisting of 50 nodes out of which 5 and 4 were submission and executing hosts respectively. A medium group mobility profile was applied to the network with $10 \mathrm{Km} / \mathrm{hour}$. The power consumption in case of the proposed heterogeneous interfaced Ad-hoc grid was evaluated against the standard Ad-hoc grid having single interface. The simulation was run for 60 minutes and the average values were calculated for 12 runs. As shown in Figure 10, the overall energy consumed by the whole network nodes is far less for heterogeneous interfaced Ad-hoc grid against the standard Ad-hoc grid. The obvious reason is that in standard Ad-hoc grid, all the nodes are using the IEEE 802.11 technology which requires more energy for per bit transmission. In the case of heterogeneous Ad-hoc grid, the power consumption is low comparatively because of the intelligent decision taken at the Interface selection layer.

\section{CONCLUSION}

The new area of Mobile ad-hoc grid is an interesting application domain of grid computing where many real life scenarios can be implemented. The integration of ad-hoc network, having nodes with multiple heterogeneous interfaces, with grid computing presents many challenges to the research community. In this paper we have first analyzed both reactive and proactive routing protocols for mobile adhoc health grid system. We were interested in quick response time of protocols and routing overhead, as systems of such an emergency nature needs very quick and reliable responses. Based on our analysis, it is concluded that DSR is the best protocol based on its quick response time for moderate to large networks with high node mobility. In small networks with low mobility GRP was found to be the best candidate routing protocol in terms of response time and routing overhead. The model was further extended to accommodate the Ad-hoc nodes having multiple heterogeneous interfaces. A thorough simulation was conducted and the comparison of the existing system was made. The results showed that Ad-hoc grid with multiple heterogeneous interfaces proved to be far more efficient in terms of energy consumption. In future we are interested in studying the complexity of the heterogeneous interfaced Ad-hoc grid and further modifying the existing model to accommodate inter-technology interface switch for throughput and connectivity enhancement and optimization.

\section{REFERENCES}

[1] D. Bruneo, M. Scarpa, A. Zaia, A. Puliafito, "Communication Paradigms for Mobile Grid Users", 3rd International Symposium on Cluster Computing and the Grid May 2003, Tokyo, Japan

[2] S. Corson and J. Macker, "Mobile Ad hoc Networking (MANET): Routing Protocol Performance Issues and Evaluation Considerations," RFC 2501, Jan. 1999

[3] H. Li, L. Sun and E.C. Ifeachor, "Challenges of Mobile ad-hoc Grids and their Applications in e-Healthcare," 2nd Int. Conf. on Computational Intelligence in Medicine and Healthcare (CIMED2005), Lisbon, Portugal.

[4] S. Jan, I. Shah and H. Al-Raweshidy, "Performance analysis of proactive and reactive routing protocols for mobile ad-hoc grid in e-health applications," in Communication Software and Networks, 2009. ICCSN'09. International Conference on, 2009, pp. 484488.

[5] Per Johansson, Tony Larsson, Nicklas Hedman “ Scenario-based performance analysis of routing protocols for mobile ad-hoc networks", International Conference on Mobile Computing and Networking Proceedings of the 5th annual ACM/IEEE international conference on Mobile computing and networking.

[6] Josh Broth David A. Maltz David B. Johnson Yih-Chun $\mathrm{Hu}$, "A Performance Comparison of Multi-Hop Wireless Ad Hoc Network Routing", International Conference on Mobile Computing and Networking Proceedings of the 4th annual ACM/IEEE international conference on Mobile computing and networking.

[7] Saniir R. Das, Cliarles E. Perkins, Elizabeth M. Roycr, "Performance Comparison of Two On-demand Routing protocols for Ad Hoc Networks", INFOCOM 2000. Nineteenth Annual Joint Conference of the IEEE Computer and Communications Societies. Proceedings. IEEE, p: 3-12 vol.1

[8] Richard Draves, Jitendra Padhye, Brian Zill, "Comparison of routing metrics for static multi-hop wireless networks". Proceedings of the 2004 conference on Applications, technologies, architectures, and protocols for computer communications. P: $133-144$, year 2004. 
[9] Cano, J.-C. Manzoni, P. "A performance comparison of energy consumption for Mobile Ad HocNetwork routing protocols". Modeling, Analysis and Simulation of Computer and Telecommunication Systems, 2000. Proceedings. 8th International Symposium on.

[10] A. Raniwala, K. Gopalan and T. Chiueh, "Centralized channel assignment and routing algorithms for multichannel wireless mesh networks," ACM SIGMOBILE Mobile Computing and Communications Review, vol. 8, pp. $50-65,2004$

[11] M. Kodialam and T. Nandagopal, "Characterizing the capacity region in multi-radio multi-channel wireless mesh networks," in Proceedings of the 11th Annual International Conference on Mobile Computing and Networking, 2005, pp. 73-87.

[12] M. Alicherry, R. Bhatia and L. E. Li, "Joint channel assignment and routing for throughput optimization in multi-radio wireless mesh networks," in Proceedings of the 11th Annual International Conference on Mobile Computing and Networking, 2005, pp. 58-72.

[13] A. Raniwala and T. Chiueh, "Architecture and algorithms for an IEEE 802.1 1-based multi-channel wireless mesh network," in INFOCOM 2005. 24th Annual Joint Conference of the IEEE Computer and Communications Societies. Proceedings IEEE, 2005,

[14] S. M. Das, H. Pucha, D. Koutsonikolas, Y. C. Hu and D. Peroulis, "DMesh: Incorporating Practical Directional Antennas in Multichannel Wireless Mesh Networks," IEEE J. Select. Areas Commun., vol. 24, pp. 2028, 2006.

[15] S. Raman, A. Ganz and R. R. Mettu, "Fair bandwidth allocation framework for heterogeneous multi-radio wireless mesh networks," in Broadband Communications, Networks and Systems, 2007. BROADNETS 2007. Fourth International Conference on, 2007, pp. 898-907

[16] H. Liu, X. Liu, C. N. Chuah and P. Mohapatra, "Heterogeneous wireless access in large mesh networks," in Mobile Ad Hoc and Sensor Systems, 2008. MASS 2008. 5th IEEE International Conference on, 2008, pp. 233-242
[17] I. Shah, S. Jan, S. Mahmud and H. Al-Raweshidy, "Optimal path discovery with mobility management in heterogeneous mesh networks," in Future Computer and Communication, 2009. ICFCC 2009. International Conference on, 2009, pp. 57-61.

[18] P Muhlethaler, T Clausen, A Laouiti, A Qayyum, L, "Optimized link state routing protocol for ad hoc networks", IEEE INMIC, 2001

[19] CE Perkins, P Bhagwat "Highly dynamic DestinationSequenced Distance-Vector routing (DSDV) for mobile computers", ACM SIGCOMM Computer Communication Review, 1994

[20] C Perkins, EM Royer, SR Das, "Ad hoc On-Demand Distance Vector (AODV) Routing”. 2003 citeseer.ist.psu.edu

[21] David B. Johnson, David A. Maltz, Yih-Chun Hu, "The Dynamic Source Routing Protocol for Mobile Ad Hoc Networks (DSR)", RFC 4738, Year 2007

[22] V. Park, S. Corson, "Temporally-Ordered Routing Algorithm (TORA)"Version1,Functional Specification, Internet Draft, IETF MANET Working Group, June 2001

[23] GRP Opnet modeler documentation, www.opnet.com

[24] J. C. Navas and T. Imielinski, "Geographic Addressing and Routing," Proc. 3rd ACM/IEEE Intn'l. Conf. Mobile Comp. Net., Budapest, Hungary, Sept. 26-30, 1997.

[25] S. Basagni et al., "A Distance Routing Effect Algorithm for Mobility (DREAM)," ACM/IEEE Int'l. Conf. Mobile Comp. Net.,1998, pp. 76-84.

[26] T Camp, J Boleng, V Davies, "A survey of mobility models for ad hoc network research". Wireless Communications and Mobile Computing, 2002

[27] standards.ieee.org/about/get/802/802.11.html Last accessed: 24th December, 2011.

[28] http://standards.ieee.org/about/get/802/802.15.html Last accessed: 24th December, 2011. 